\title{
The Wave-Front Correction System for the Sunrise Balloon-Borne Solar Observatory
}

\author{
T. Berkefeld · W. Schmidt • D. Soltau • A. Bell • H.P. Doerr • B. Feger • R. Friedlein • \\ K. Gerber · F. Heidecke · T. Kentischer • O. v. d. Lühe · M. Sigwarth · E. Wälde • \\ P. Barthol · W. Deutsch · A. Gandorfer · D. Germerott • B. Grauf • R. Meller • \\ A. Álvarez-Herrero • M. Knölker • V. Martínez Pillet · S.K. Solanki · A.M. Title
}

Received: 8 June 2010 / Accepted: 4 November 2010 / Published online: 8 December 2010

(C) The Author(s) 2010. This article is published with open access at Springerlink.com

\begin{abstract}
This paper describes the wave-front correction system developed for the Sunrise balloon telescope, and it provides information about its in-flight performance. For the correction of low-order aberrations, a Correlating Wave-Front Sensor (CWS) was used. It consisted of a six-element Shack - Hartmann wave-front sensor (WFS), a fast tip-tilt mirror for the compensation of image motion, and an active telescope secondary mirror for focus correction. The CWS delivered a stabilized image with a precision of $0.04 \operatorname{arcsec}(\mathrm{rms})$, whenever the coarse pointing was better than \pm 45 arcsec peak-to-peak. The automatic focus adjustment maintained a focus stability of 0.01 waves in the focal plane of the CWS. During the 5.5 day flight, good image quality and stability were achieved during 33 hours, containing 45 sequences, which lasted between 10 and $45 \mathrm{~min}$.
\end{abstract}

HAO/NCAR is sponsored by the National Science Foundation.

T. Berkefeld $(\varangle) \cdot$ W. Schmidt · D. Soltau · A. Bell · H.P. Doerr · B. Feger · R. Friedlein · K. Gerber ·

F. Heidecke · T. Kentischer · O. v. d. Lühe · M. Sigwarth · E. Wälde

Kiepenheuer-Institut für Sonnenphysik, Schöneckstraße 6, 79104 Freiburg, Germany

e-mail: berke@kis.uni-freiburg.de

P. Barthol · W. Deutsch · A. Gandorfer · D. Germerott · B. Grauf · R. Meller · S.K. Solanki

Max-Planck-Institut für Sonnensystemforschung, Max-Planck-Straße 2, 37191 Katlenburg-Lindau, Germany

V. Martínez Pillet

Instituto de Astrofísica de Canarias, C/Via Láctea, s/n, 38205 La Laguna (Tenerife), Spain

M. Knölker

High Altitude Observatory, P.O. Box 3000, Colorado 80301, USA

A.M. Title

Lockheed Martin Solar and Astrophysics Laboratory, Bldg. 252, 3251 Hanover Street, Palo Alto, CA 94304, USA

A. Álvarez-Herrero

Instituto Nacional de Técnica Aeroespacial, 28850 Torrejón de Ardoz, Madrid, Spain 
Keywords Adaptive optics · Balloon · Image stabilization · Instrumentation ·

Shack-Hartmann · Tip-tilt correction · Wavefront sensing

\section{Introduction}

The Sunrise ${ }^{1}$ balloon-borne telescope for solar observations performed its first science flight in June 2009 on a NASA long-duration balloon flight from Kiruna, Sweden to Somerset Island in North-East Canada (see Barthol et al., 2010; Schmidt et al., 2010). After a perfectly smooth launch, Sunrise headed West, across the Northern Atlantic and Greenland. About 1.8 TByte of science data were collected. A few days after landing, the data disks, the telescope and the gondola were safely recovered. The processing of the scientific and engineering data is underway, as well as an inspection of the flight hardware.

Stratospheric balloon-borne telescopes have two fundamental advantages over groundbased telescopes: they permit UV observations and they provide a seeing-free image quality over the full field of view (FoV). However, pointing to the Sun and tracking a feature on the solar surface is a formidable task, especially for a telescope hanging under a balloon that is driven by stratospheric winds at an altitude of $36 \mathrm{~km}$. In addition to the apparent (diurnal and seasonal) motion of the Sun, there is a number of oscillatory modes that may be induced by variable winds in the stratosphere, and taken up by the balloon - gondola system. The tip-tilt correction of CWS was built with a range of \pm 45 arcsec, and a closed-loop bandwidth of $60 \mathrm{~Hz}$ ( $6 \mathrm{~dB}$ attenuation of tip-tilt). The instrument worked reliable throughout the flight. Closed-loop operations were possible, whenever the gondola pointing was within the angular range of the CWS tip-tilt mirror.

Section 2 explains the optical design, Section 3 deals with the wave-front correction algorithms. The electronics and tip-tilt hardware is described in Section 4. The control software is addressed in Section 5, followed by a discussion of the in-flight performance in Section 6. Finally, Section 7 concludes this paper.

\section{Opto-Mechanical Design}

Sunrise is a $1 \mathrm{~m}$ Gregory-type telescope (see Figure 1 and Barthol et al. (2010) for a detailed description). The parabolic primary mirror M1 (f/2.42) provides a full disk image in its focal plane. Here a field stop which is also a heat rejection device reflects $99 \%$ of the sunlight. Only a field of view of 200 arcsec passes to the elliptical secondary mirror M2, which delivers a f/24 focus to F2.

Sunrise feeds two scientific instruments: SuFI (Sunrise Filter Imager, see Gandorfer et al., 2010), an UV-imager, and IMaX (Imaging Magnetograph Experiment, see Martínez Pillet et al., 2010), a compact filter based magnetograph. In addition, the CWS (Correlating Wave-Front Sensor) is fed by Sunrise to measure the image shift and possible loworder aberrations (focus and coma), which are due to misalignment of M1 or M2 caused by thermo-elastic deformations of the telescope during flight.

\footnotetext{
${ }^{1}$ Sunrise is an international collaboration of the Max-Planck Institut für Sonnensystemforschung (KatlenburgLindau, Germany), the High Altitude Observatory (Boulder, U.S.), the Lockheed-Martin Solar and Astrophysics Laboratory (Palo Alto, U.S.), the IMaX Consortium (Tenerife, Granada, Madrid, Spain) and the Kiepenheuer-Institut für Sonnenphysik (Freiburg, Germany).
} 
Figure 1 Optical design of the Sunrise telescope.
F2
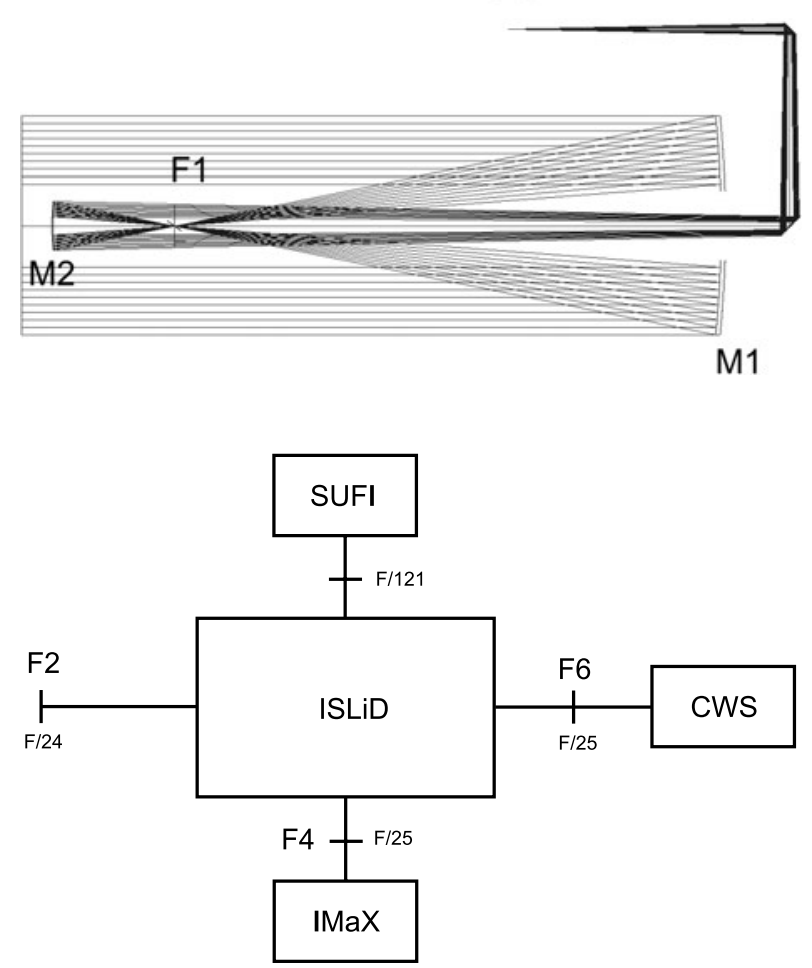

Figure 2 Scheme of the Image Stabilization and Light Distribution system (ISLiD).
For tip-tilt correction, a pupil image of appropriate size is needed where the tip-tilt mirror can be placed. The mirror size determines the necessary mirror stroke (the smaller the mirror the larger the stroke). On the other hand a larger pupil means a longer focal length. The optical solution which was chosen is a field lens which provides a $35 \mathrm{~mm}$ pupil image. Here the tip-tilt mirror M105 (notation compatible with official Sunrise documentation) was placed. This mirror is part of the ISLiD (Image Stabilization and Light Distribution) system (see Gandorfer et al., 2010) and is further described in Section 4. While maintaining the diffraction-limited image quality, ISLiD provides the following:

- the desired f/121 image for the UV channel (SuFI);

- an f/25 image for IMaX (visible);

- an $\mathrm{f} / 25$ image to the CWS (visible);

- a possibility to feed a third instrument (near-IR spectrograph, planned for the second flight).

The main functions of ISLiD are shown schematically in Figure 2. For this purpose, a modified Schwarzschild system is used to magnify the image for SuFI. Four relay lenses demagnify the image and shift the pupil so that IMaX and the CWS are illuminated with an f/25 beam, with a pupil at infinity. An additional correction element accounts for the beam splitter not used in collimated light.

The CWS is a Shack-Hartmann ( $\mathrm{SH})$ type wave-front sensor working at $500 \mathrm{~nm}$. Its optical design is shown in Figure 3. It picks up the image at the entrance focus F6, which also defines the system focus of Sunrise. Differential foci between the science instruments 


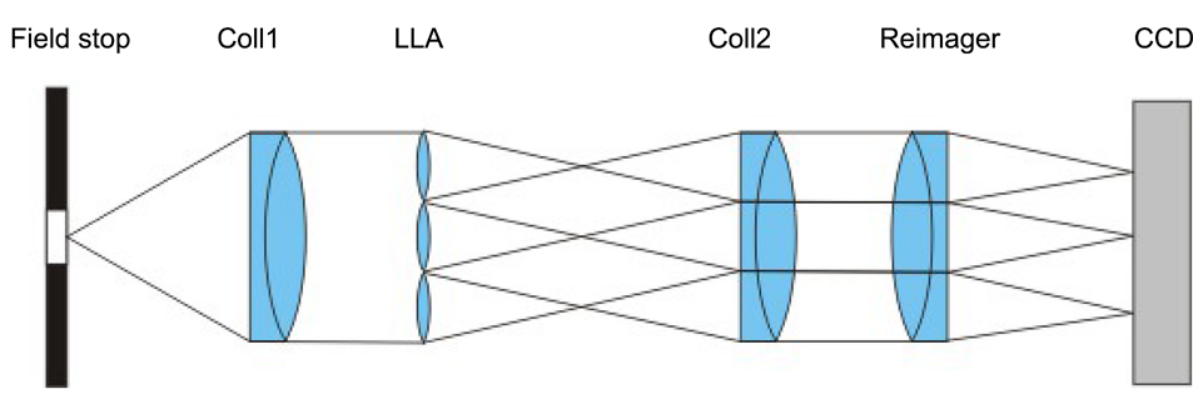

Figure 3 Scheme of the wave-front sensor of Sunrise. The field stop coincides with a focal plane of the telescope.

Figure 4 Illumination pattern of the CWS lenslet array. The image of the $1 \mathrm{~m}$ entrance pupil provides a homogeneous illumination of the six peripheral micro-lenses, except for the (small) influence of the spiders. The central lenslet is obscured by the secondary mirror and is not used.

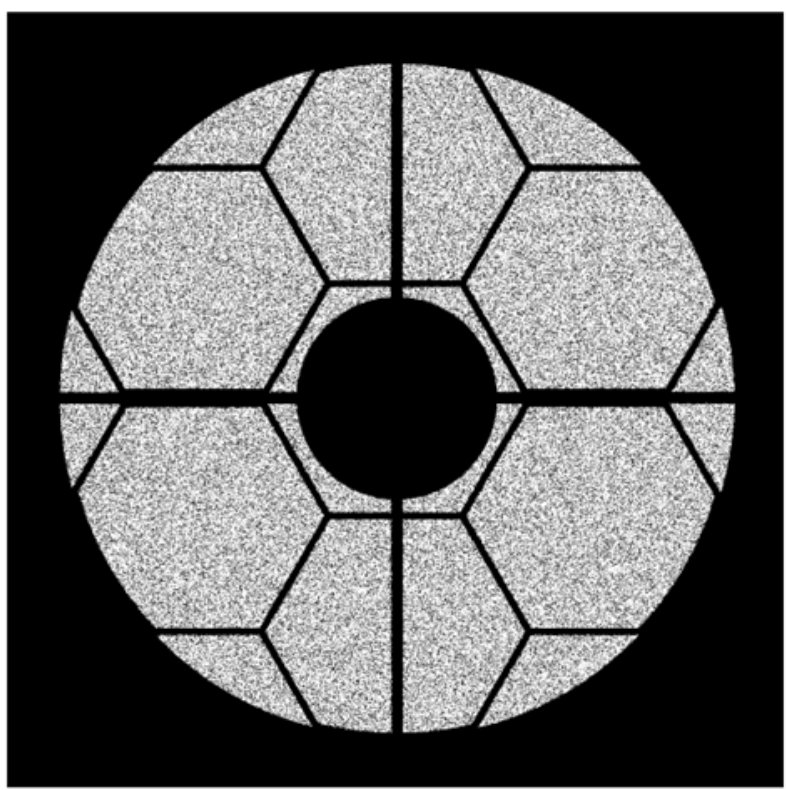

and the CWS are not corrected by the CWS and have been avoided by a careful system alignment. A field stop with a square area of $1.54 \times 1.54 \mathrm{~mm}^{2}$ limits the field of view to 13 arcsec. Coll1 $(f=100 \mathrm{~mm})$ images a $4.6 \mathrm{~mm}$ pupil on a hexagonal lenslet array (LLA, $f=81 \mathrm{~mm}$ ) which consists of seven hexagonal micro-lenses. The geometry of the lenslet array is shown in Figure 4. The central one is obscured by the shadow of the telescope's secondary. A relay lens system consisting of a collimator Coll2 $(f=160 \mathrm{~mm})$ and an imaging lens $(f=90 \mathrm{~mm})$ picks up the focal plane of the LLA and images the subfields on the wave-front sensor camera. The image scale is chosen so that $1 \operatorname{arcsec} c o r r e s p o n d s$ to five pixels in the camera plane. The left panel of Figure 15 in Section 6 shows the six images on the high-speed camera. It is an original flight picture taken as a screen copy from the ground control system. Solar granulation is clearly visible at high contrast and good image sharpness. 


\section{Wave-Front Correction}

\subsection{Overview}

An overview of the CWS architecture is given in Figure 5 where black lines are communication paths that are typically labeled with the interface used. The upper region shows the light path from the entrance pupil (EP) of the telescope to the camera. CWS electronics components are shown as yellow boxes. In addition, the figure shows the pointing system (PS, green box) provided by the High Altitude Observatory in Boulder, U.S., and the main telescope controller (MTC, pink box) delivered by the manufacturer of the telescope, Kayser Threde in Munich, Germany. The actual wave-front correction is controlled by a computer named CW-AO depicted in the right part of the overview.

The main components of the wave-front correction system are the following devices: the Shack - Hartmann wave-front sensor (Section 2), a high-speed camera (Section 4.5), the control loop computer that converts the shifts measured with the SH sensor into actuator signals (Section 4.2), the fast tip-tilt mirror (1700 Hz update rate, Section 4.4) and the slow secondary mirror M2 (0.1 Hz update rate) (see Schmidt et al., 2004, 2006). The telescope secondary, M2, has tight alignment tolerances with respect to M1, therefore M2 is mounted on a motorized $x y z$ stage that is controlled by the CWS to ensure proper alignment. Lateral ( $x y)$ misalignment causes coma and image shift, while axial $(z)$ misalignment causes spherical aberrations (negligible) and defocus.

\subsection{Algorithms}

The Shack-Hartmann wave-front reconstruction is done in much the same way as in the solar AO system KAOS at the Vacuum Tower Telescope on Tenerife (see von der Lühe et al., 2003).

The data reduction consists of the following steps for each $64 \times 64$ pixel sub-aperture, in the order given below:

- dark/flat correction;

- subtraction of the average (of this sub-aperture) intensity;

- removal of the intensity gradient across the FoV;

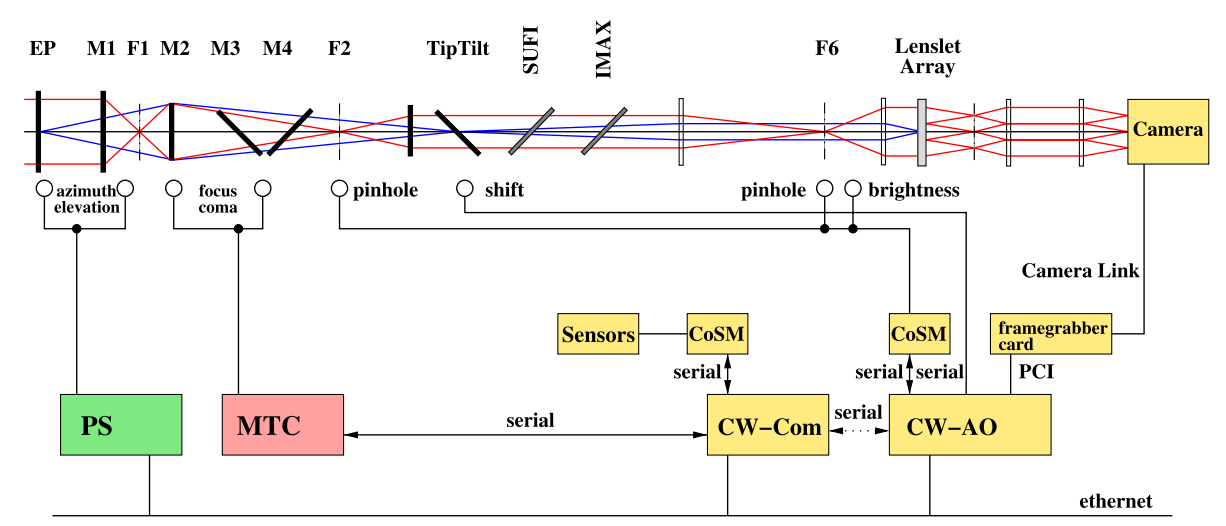

Figure 5 Scheme of the Sunrise CWS hardware and communication lines. CWS electronics components are shown as yellow boxes, PS denotes the gondola pointing system and MTC the main telescope controller. 
- application of a Hamming window $W(x, y)=w(x) \cdot w(y)$ with $w(i)=0.54+(0.54-1)$. $\cos (2 \pi \cdot i /(64-1))$;

- addition of the former average intensity;

- intensity normalization.

The subtraction and addition of the average intensity is advisable for the application of the Hamming window which eliminates aliasing problems of the FFT-based cross correlation. The removal of the intensity gradients and the intensity normalization are required to get the correct position of the correlation peak.

The reduced sub-aperture images are now cross correlated against a cross correlation reference sub-aperture image that has undergone the same data reduction. We use FFTbased cross correlations, since they are more efficient than direct cross correlations when the number of pixels in the correlation field is large. The correlation function $C$ of sub-aperture $S$ and reference $R$ can be calculated as

$$
C=\mathrm{FFT}^{-1}\left[\mathrm{FFT}[S] \cdot(\operatorname{FFT}[R])^{*}\right],
$$

the asterisk denoting the complex conjugate. We apply a $3 \times 3$ pixel 2D-parabolic fit around the brightest pixel to calculate the position of the correlation maximum $C_{\max }$. The $x y$-shift $S_{\text {shift }}$ is the error signal of the WFS:

$$
S_{\text {shift }}=R_{\text {ref }}-S_{\text {ref }}+R_{\text {shift }}+C_{\text {max }}-64 / 2,
$$

$R_{\text {ref }}$ and $S_{\text {ref }}$ being the spot positions of the perfect wave-front of cross correlation reference image $R$ and sub-aperture $S$. These are obtained by moving a pinhole into the entrance focus of the WFS and measuring the pinhole image positions on the SH detector.

Since the solar structures evolve, the cross correlation reference image has to be updated regularly. The update rate must be shorter than the time scale of the evolution of solar structures resolved by a sub-aperture, and has therefore been set to $0.2 \mathrm{~Hz}$ (every $5 \mathrm{~s}$ ).

The $x y$-shift $R_{\text {shift }}$ of the cross correlation reference image propagates in the same way:

$$
R_{\text {shift,new }}=R_{\text {shift,old }}+C_{\max }-64 / 2 \text {. }
$$

\subsection{Wavefront Reconstruction}

The aberrations that need to be sensed determine the number of Shack-Hartmann subapertures.

- The tip-tilt needs to be corrected in closed loop.

- Defocus (caused by M2 or M3/M4 axial misalignment) needs to be corrected in closed loop (focus drifts due to temperature changes).

- Coma (caused by M2 lateral misalignment), in principle, could have been removed in closed loop by a lateral movement of M2. However, since a lateral movement of M2 produces 400 waves of tip-tilt for each wave of coma, ${ }^{2}$ a closed-loop coma-correction would have had a negative impact on the tip-tilt performance. Instead, the coma was corrected prior to the flight. Since the initial alignment of the telescope was very good, only slight lateral M2 adjustments had to be made, so that no vignetting of the F2 field stop by the F1 field stop occurred. During the flight, coma was measured only.

\footnotetext{
${ }^{2}$ One wave $(500 \mathrm{~nm})$ rms of tip-tilt aberration corresponds to 0.37 arcsec of image shift.
} 
- Spherical aberration (SA, caused by M2 axial misalignment): although it is in principle possible to remove both defocus and SA simultaneously with M2 and M3/M4, an analysis of the optical errors showed that the amount of spherical aberration induced by a defocus of M2 is negligible. Therefore it was sufficient to statically align M2 and M3/M4 prior to the flight and correct the in-flight defocus with M2 only. SA does not need to be sensed.

- There are other higher-order errors (such as triangular coma due to the M1 support). Interferometric measurements have shown that they have only minor contributions to the overall wavefront error; furthermore, there is no degree of freedom for correction anyway. Higher-order modes need not be sensed.

We concluded that a six sub-aperture WFS is sufficient to sense tip-tilt, defocus and coma.

For sensing these five modes, a simple reconstruction is sufficient. The reconstruction matrix for the tip-tilt mirror is the SVD-inverted measured interaction matrix between $x y$ shifts and the actuators. The tip-tilt servo is either of PI (proportional + integral) or PID (proportional + integral + differential) type. The reconstruction matrix for M2 has been pre-calculated via Zernike polynomials. Since the focus drift due to changing zenith angles occurs on timescales of minutes, M2 receives a time-averaged (over $10 \mathrm{~s}, 17000$ frames) signal, where a pure proportional controller is sufficient. During the flight, only tip-tilt and focus have been corrected in closed loop.

\subsection{Real Time Control}

In order to achieve a high tip-tilt system bandwidth, the delays in the control loop have to be as small as possible. Listed below are the delays of the correction:

- $290 \mu \mathrm{s}=1 / 2$ of the inverse of the frame rate;

- $130 \mu \mathrm{s}=$ readout time after which the first two sub-aperture images have been transferred into the frame buffer of the real time computer, and the calculation starts (the total readout time is $380 \mu \mathrm{s}$ );

- $570 \mu \mathrm{s}=$ calculation time

(190 $\mu$ s per sub-aperture per CPU, 2 CPUs);

- $120 \mu \mathrm{s}=$ output + DA conversion;

- $200 \mu \mathrm{s}=\mathrm{HV}$ rise time;

- $200 \mu \mathrm{s}=$ tip-tilt mirror settling time.

An additional $290 \mu$ s sample and hold has to be added to the total delay of ca. $1.5 \mathrm{~ms}$ between the occurrence of a disturbance and its correction. This results in a closed loop $6 \mathrm{~dB}$ bandwidth of $60 \mathrm{~Hz}$ for the PID controller and $35 \mathrm{~Hz}$ for the PI controller, overachieving the $30 \mathrm{~Hz}$ bandwidth specification. Figure 6 shows the disturbance attenuation as a function of the frequency for the PID controller.

The real time control loop software is a variant of the KAOS-system that is used at the Vacuum Tower Telescope on Tenerife (Berkefeld, 2007). In order to achieve a compute time of only $190 \mu$ s per sub-aperture per CPU for a $64 \times 64$ pixel cross correlation field, the compute intensive parts of the code (data reduction and cross correlation) had to be handoptimized for the Altivec vector processing unit of the Motorola G4 CPUs.

\subsection{Measurement Accuracy}

The measurement accuracy of a correlating SH sensor depends on the following properties: 


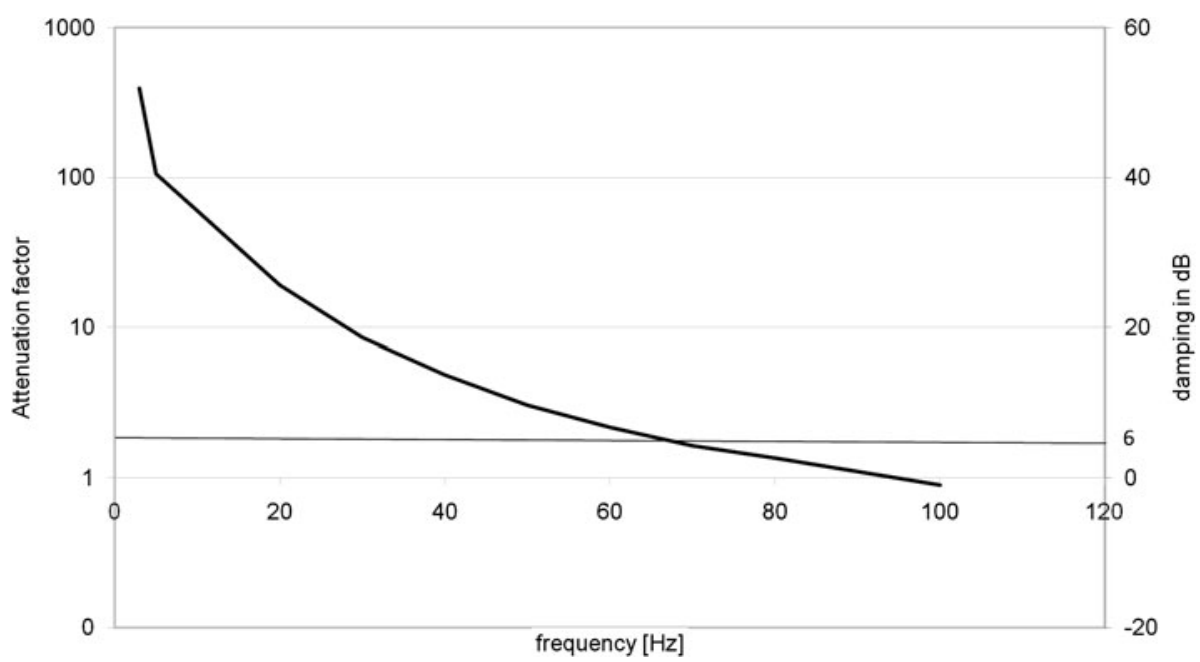

Figure 6 Performance of the tip-tilt correction system, as measured in the laboratory. The $6 \mathrm{~dB}$ point, corresponding to a correction of a factor of two, is marked as a horizontal line. The corresponding closed-loop frequency is $60 \mathrm{~Hz}$.

- image contrast and its (spatial) power spectrum (signal);

- sub-aperture diameter;

- observing/sensing wavelength;

- pixel scale;

- number of pixels per cross correlation field;

- noise (typically shot noise).

The Sunrise SH sensor works at $500 \mathrm{~nm}$ and uses telescope sub-apertures of $0.33 \mathrm{~m}$, yielding a spatial resolution of $0.4^{\prime \prime}$. In order to take advantage of this high (for a $\mathrm{SH}$ sensor) resolution, we use a pixel scale of $0.2^{\prime \prime}$ pixel $^{-1}$ and $64 \times 64$ pixels for the cross correlation $\left(12.8^{\prime \prime}\right.$ field of view). For calculating the rms intensity contrast as seen by the wave-front sensor, the original power spectrum of the solar granulation has to be multiplied by the modulation transfer function of a sub-aperture. This reduces the original $8.5 \% \mathrm{rms}$ granulation contrast, as measured with the IMaX-instrument, to $6 \%$, as seen by the SH sensor.

Michau, Rousset, and Fontanella (1992) estimated that the measurement noise variance $\sigma_{\mathrm{snr}}^{2}\left(\right.$ in $\lambda^{2}$ ) for a critically sampled, correlating single aperture is

$$
\sigma_{\mathrm{snr}}^{2}=\frac{20 \sigma_{\text {noise }}^{2}}{n^{2} \sigma_{\text {signal }}^{2}},
$$

where $\sigma_{\text {noise }}$ denotes the rms noise level, $n$ the number of pixels across the cross correlation field of view (64) and $\sigma_{\text {signal }}$ the image contrast. The noise level has two main contributors: for the photon noise we assume a typical use of $160000 \mathrm{e}^{-}$(photo electrons) per pixel ( $80 \%$ full well capacity), yielding $400 \mathrm{e}^{-}$. The 8-bit digitization leads to a noise of $200000 \mathrm{e}^{-} / 256=800 \mathrm{e}^{-}$peak-to-peak, which corresponds to about $270 \mathrm{e}^{-} \mathrm{rms}$. The total noise level is therefore $480 \mathrm{e}^{-}$. After applying Equation (1) and subsequent conversion from a wave-front error (in $\lambda$ ) to a pointing (tip-tilt error, in arcsec), the tip-tilt measurement error for a single $33 \mathrm{~cm}$ sub-aperture is $\sigma_{\text {tip-tilt,single }}=0.005^{\prime \prime} \mathrm{rms}$ (per axis). Averaging over six 
sub-apertures finally results in

$$
\sigma_{\text {tip-tilt,total }}=0.002^{\prime \prime} \mathrm{rms} \text {. }
$$

\section{Hardware}

The CWS electronics consists of two main parts. The main electronics unit (MEU) contains the control processor and the processor for the wave-front correction, as well as all communication and thermal control hardware. The MEU is located outside the telescope, in the shadow of the solar panels, in order to minimize any thermal disturbance to the light path, and in order to avoid external heating by sunlight. The proximity electronics box (PEB) drives the high-speed tip-tilt mirror and is therefore located close to that mirror.

In order to qualify the hardware for the near vacuum conditions of the stratosphere, we tested the complete CWS (see Figure 7) and its electronics at the Instituto Nacional de Técnica Aeroespacial (INTA), Madrid, Spain, at pressures down to 100-200 Pascal and temperatures between $+40^{\circ}$ and $-50^{\circ}$ Celsius. No problems were found during these tests.

Figure 7 The wave-front sensor, mounted on its light-weight carbon fiber sandwich plate. On the sides, two fixation points of the isostatic mounting are visible.

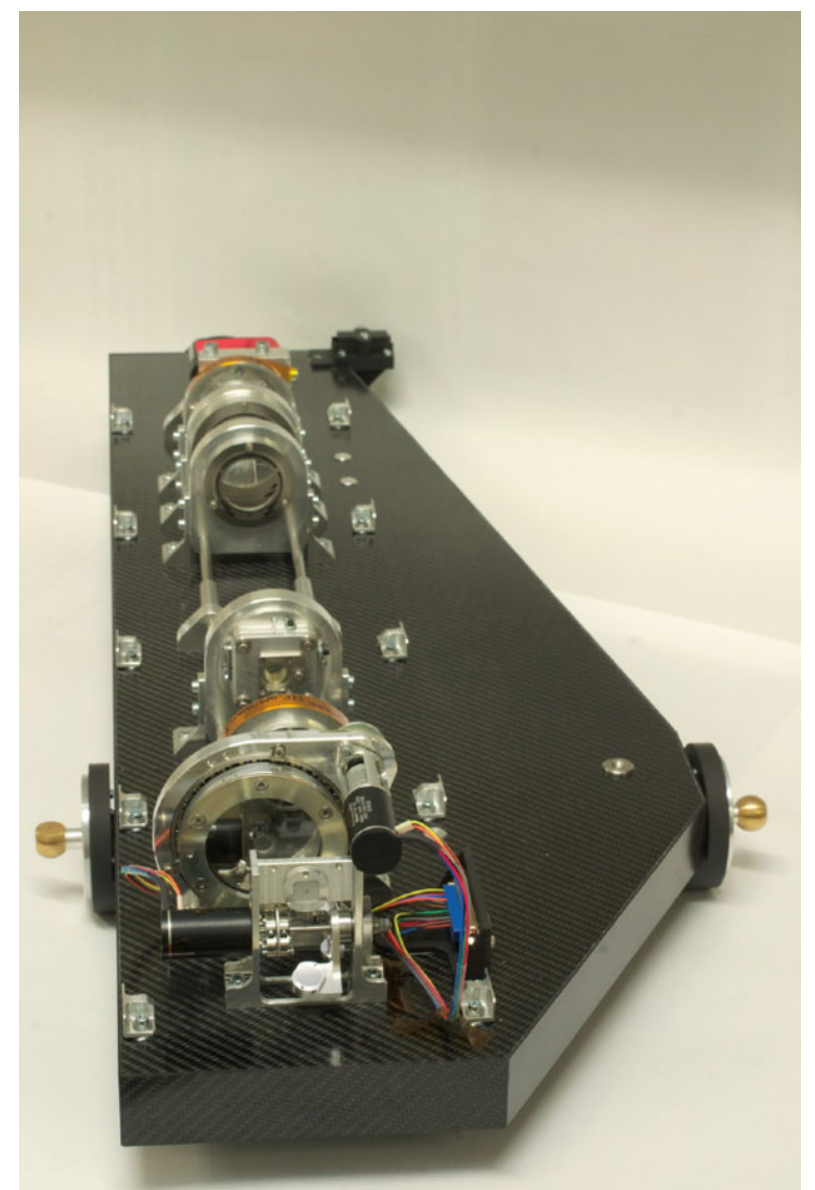




\subsection{Thermal and Environmental Design}

At flight altitude the atmospheric pressure is about 5 mbar. To dissipate the power effectively by thermal radiation, the cooling elements have an allocated radiation area, and the cooling design has to ensure that the heat is transmitted to that area. For the CW-electronics-unit we decided to work without a pressurized box, and to dissipate the power only by conduction and radiation. All electronics were designed for low power consumption and small power density to decrease or even avoid any hot spots. The advantages of a conduction cooled electronics unit in contrast to a pressurized box are reduced weight and an uncomplicated temperature control with heaters and radiation coolers. Furthermore, a pressurized box and electronics designed to work under such pressure pose the risk of failure due to loss of air pressure. To protect all components from environmental influences, we applied an electrical isolation and hydrophobic coating to all boards. The cooling elements had a Lord Aeroglaze A 276 coating with high emissivity and low absorption. The power consumption of the $\mathrm{CW}$ electronics unit in the operating mode is nearly $70 \mathrm{~W}, 45$ of which are used by the real time computer doing the wave-front correction.

The rapid fluctuations in the temperature profile in Figure 8 show the varying power consumption during the operating and non-operating phases. The low-frequency pattern shows temperature changes between day and night. The decreasing temperature at the beginning results from the ascent of the balloon and the passage through cold atmospheric layers. The noticeable peak on June, 11th coincides with the passage of the gondola over Greenland. The high albedo of the ice led to an increase of the temperature. One of the components of the CWS that required special treatment in order to operate properly at float altitude, was the high-speed camera. We designed a cooling element that connected the hot spot in the interior to the walls of the housing. Figure 9 shows an infrared image of the camera electronics before the installation of the cooling element.

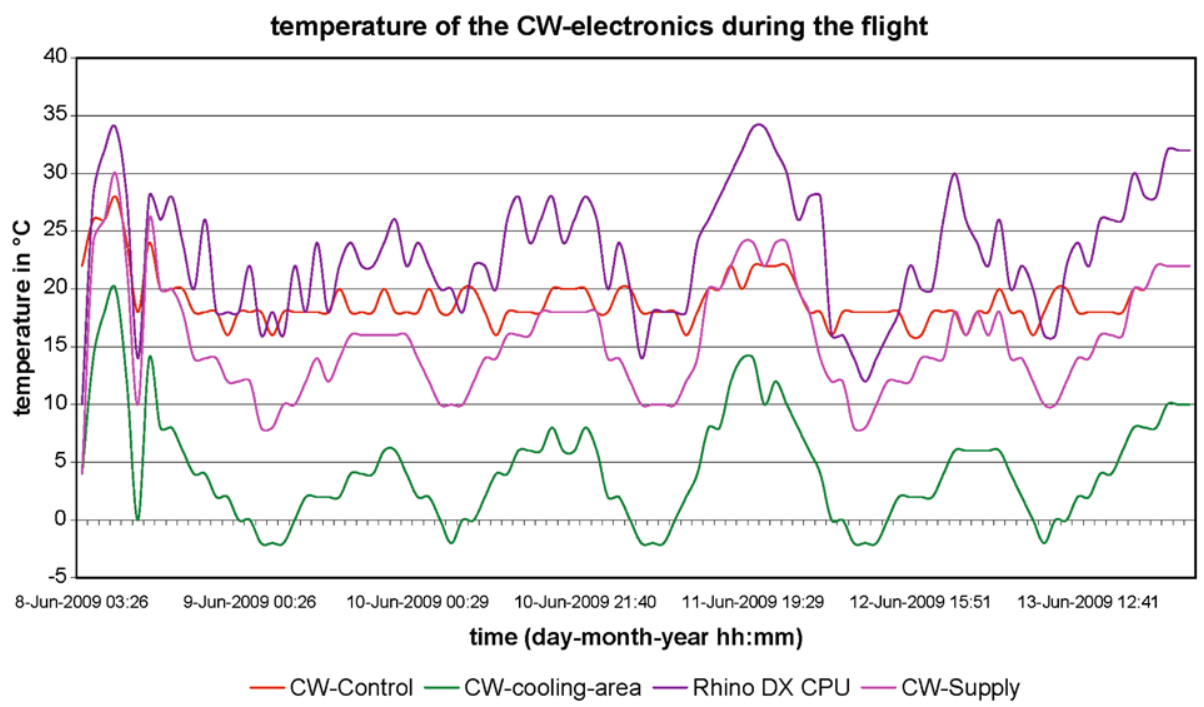

Figure 8 Temperature profile of the Main Electronics Box during the balloon flight. The slow variations correspond to temperature changes caused by the varying elevation of the Sun. The strong peak on 11 June occurred during the passage over Greenland, where sunlight was reflected toward the instruments. 
Figure 9 Infrared image of the electronics boards of the high-speed CMOS camera, prior to the installation of the conductive cooling elements. A hot spot is clearly visible.

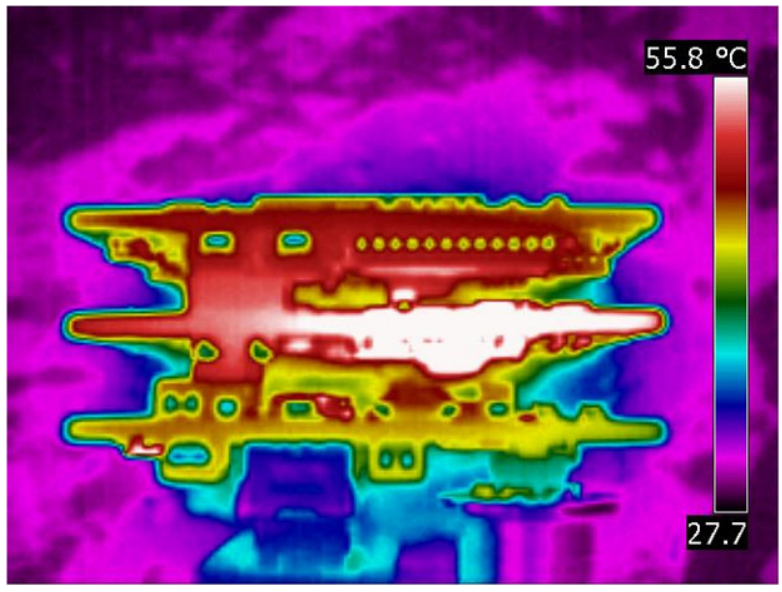

\subsection{Main Electronics Unit}

The CWS main electronics unit (Figure 10) contains three different components. The CWControl (a.k.a. CW-Com) with its A9M9750 module based on a NetSilicon's, $200 \mathrm{MHz}$, NS9750 micro-controller is a compact low energy solution from DIGI. It dissipates nearly $1.7 \mathrm{~W}$ in operating mode. The $\mathrm{CW}$-Control includes also the clients of the CoSM-Bus (Volkmer et al., 2003). This bus handles the control data of the motion units. The CW-AO is the real time computer that handles the wavefront correction control loop. It consists of the Rhino DX Board, a conduction-cooled VME single board computer with two $1 \mathrm{GHz}$ Motorola 7457 processors, made by Curtiss Wright, the frame grabber and an interface board, which is specifically designed for the Rhino DX requirements. Attached to CW-AO by two serial interfaces are the digital-to-analog converters of the tip-tilt mirror. Also serially attached to CW-AO are the mechanisms that allow changing between dark stop, field stop and pinhole at the F2 and F6 foci, and the neutral density filter that keeps the WFS camera illuminated appropriately. The power for all components of the CWS is provided from the CW-Supply, which has five voltage levels with different requirements on current or ripple. The power management is controlled by the CW-Control; it permits start up and power down of the whole system in a given order.

\subsection{Proximity Electronics Box}

The tip-tilt mirror (M105) is driven by the high-voltage amplifier of the proximity electronics box (PEB); see Figure 11. It requires two variable channels and one fixed voltage. Each variable amplifier channel consists of a DA converter with serial interface, a pre-amplifier and a push-pull power stage. The CW-AO dual processor board transmits the data to the high-voltage amplifier via two RS422 serial interfaces.

The motor controller and the high-voltage amplifier are assembled in the CWS PEB that is cooled by conduction. All electronic parts with power losses higher than $250 \mathrm{~mW}$ are treated with thermal filler and thereby coupled to the housing. A radiator outside the PEB removes the heat produced by the CWS proximity box. During the flight, the temperatures in the box were between $-10^{\circ} \mathrm{C}$ and $+20^{\circ} \mathrm{C}$. 


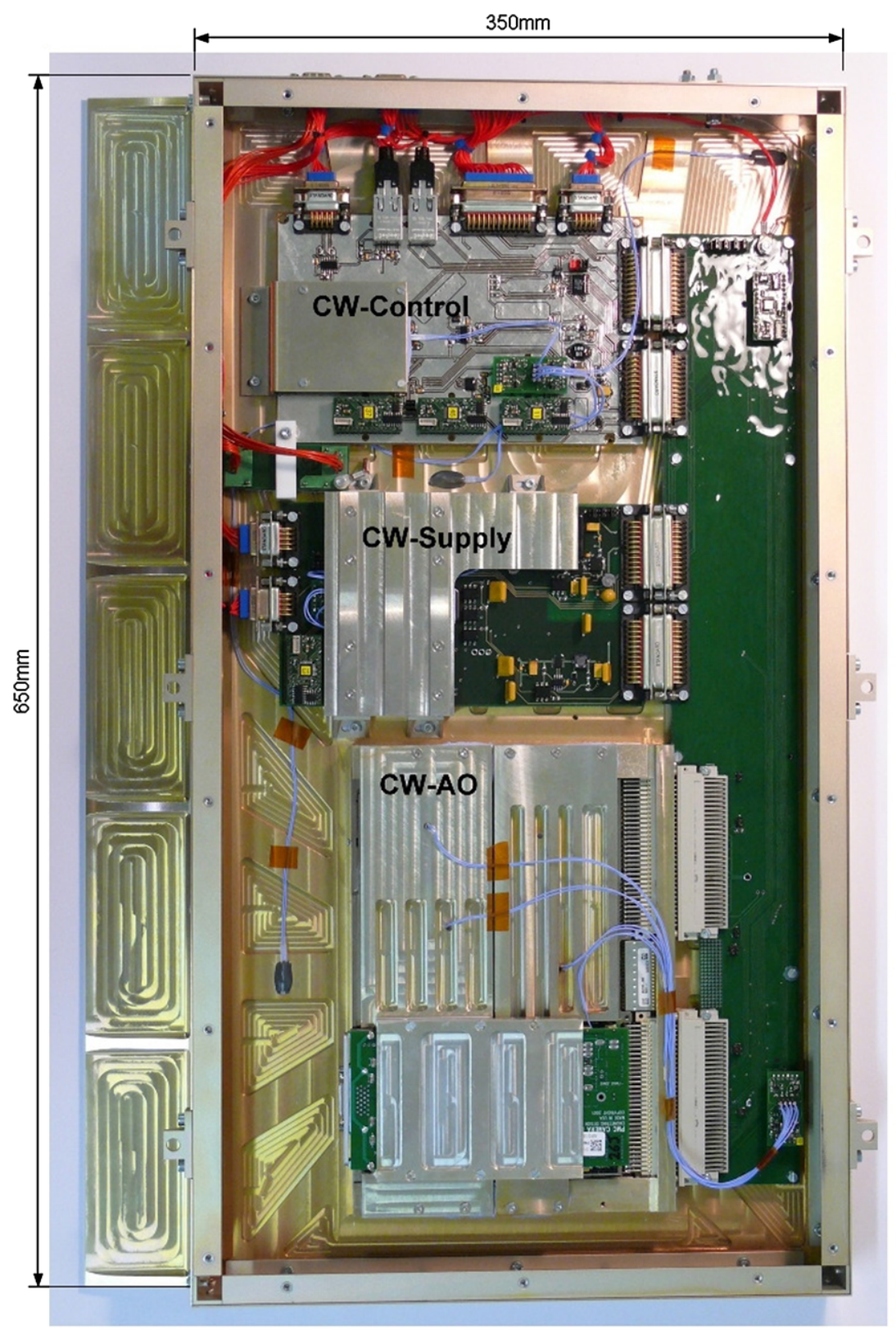

Figure 10 Main electronics box of the CWS. 'CW-Control' denotes the control processor (below the cooling plate), the main power supply is labeled 'CW-Supply', 'CW-AO' is the control loop real time computer (dual Motorola 7457 CPUs). 


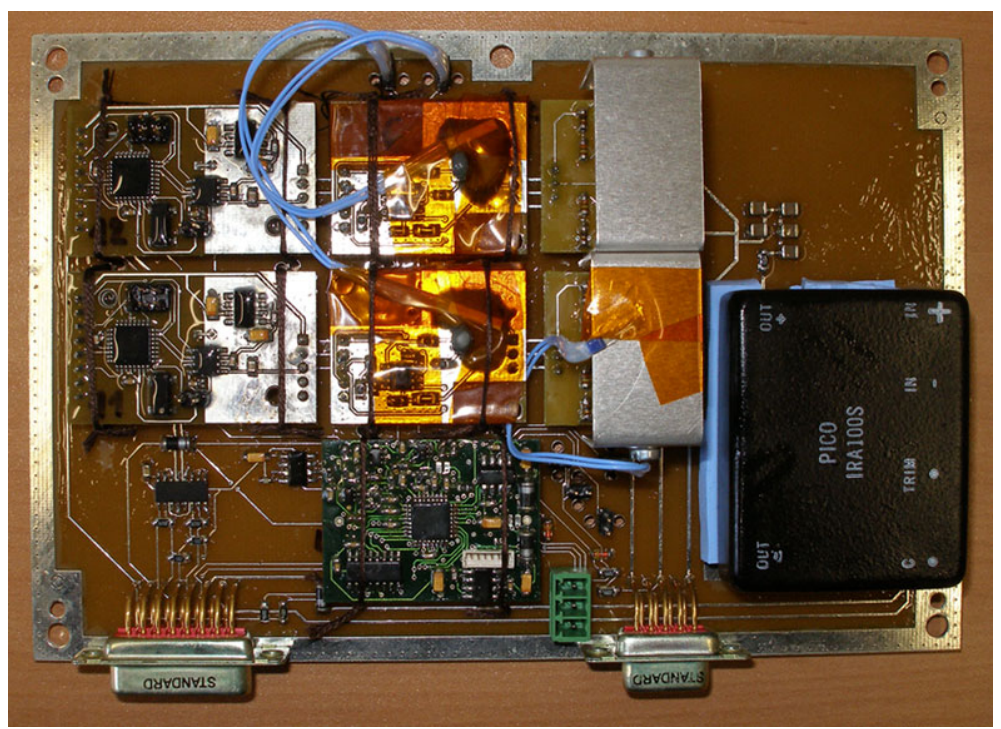

Figure 11 High-voltage amplifier for the piezo drive of the tip-tilt mirror. From left to right: serial interface with digital/analog converter, pre-amplifier, main amplifier. The green board at the bottom is a CoSM sensor server used for temperature measurements. The black box is the $100 \mathrm{~V}$ power supply for the main amplifier.

Table 1 CWS proximity electronics

\begin{tabular}{ll}
\hline Component & Characteristics \\
\hline M105 channel 1 & $7 \mathrm{~V} \ldots 93 \mathrm{~V}$ \\
M105 channel 2 & $7 \mathrm{~V} \ldots 93 \mathrm{~V}$ \\
M105 channel 3 & $100 \mathrm{~V}$ fixed \\
High-voltage supply & $18 \mathrm{~V} \ldots 36 \mathrm{~V} \rightarrow 100 \mathrm{~V}$ (max. 10 W) \\
Push-pull power stage & max. $2 * 5 \mathrm{~W} \mathrm{@60} \mathrm{Hz} \mathrm{full} \mathrm{scale} \mathrm{driven}$ \\
Pre-amplifier & $0 \mathrm{~V} \ldots .5 \mathrm{~V} \rightarrow 0 \mathrm{~V} \ldots 100 \mathrm{~V}$ \\
DA converter interface & $230.4 \mathrm{kbit} \mathrm{s}^{-1} 8 \mathrm{~N} \mathrm{1 \textrm {UART }}$ \\
DA converter update & max. $10 \mathrm{kHz}$ \\
DA converter resolution & $16 \mathrm{bit}$ \\
High-voltage scale & $97.6 \mathrm{~V} / 65535=1.489 \mathrm{mV} / \mathrm{LSB}$ \\
Dynamic amplifier range & $86 \mathrm{~V}$ \\
Tip-tilt range (on sky) & 99 arcsec \\
Tip-tilt resolution (on sky) & $0.00173 \mathrm{arcsec}$ \\
\hline
\end{tabular}

\subsection{Tip-Tilt Mirror}

For the tip-tilt correction a two axis piezo ceramic actuator from Physik Instrumente was used (P.I. S-330K065). This piezo drive fulfills all requirements for the Sunrise image stabilization (high acceleration, fast response to set points and small increments). The piezo actuators have a total tilt range of $10 \mathrm{mrad}$ (corresponding to $\pm 45 \operatorname{arcsec}$ in the sky). The minimal step size corresponds to $0.15 \mu \mathrm{rad}$ ( 1.7 mas on the sky). The dynamic characteristics are also shown in Table 1 . The piezo actuators have a very low power dissipation. The zerodur tip-tilt mirror attached to the actuator is $35 \mathrm{~mm}$ in diameter with a thickness of 
Figure 12 Tip-tilt mirror with the piezo stage (in the polished cylinder) and the mechanical interface to the mount assembly.

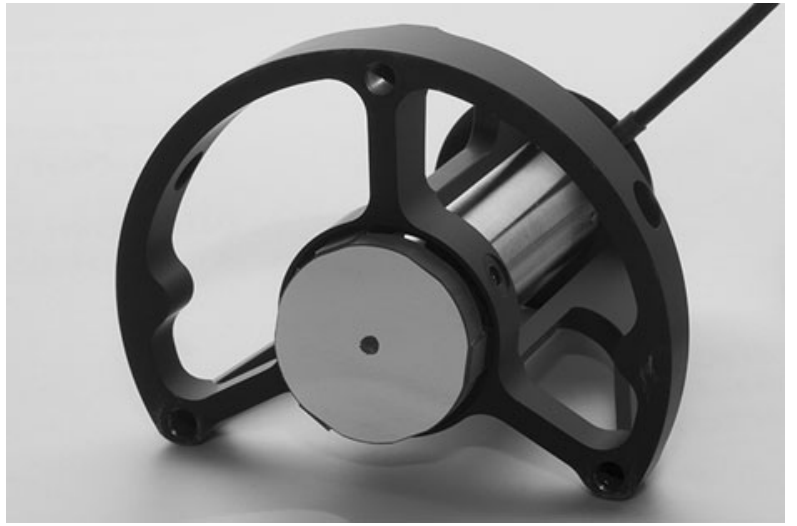

Excitation of the tip tilt mechanism

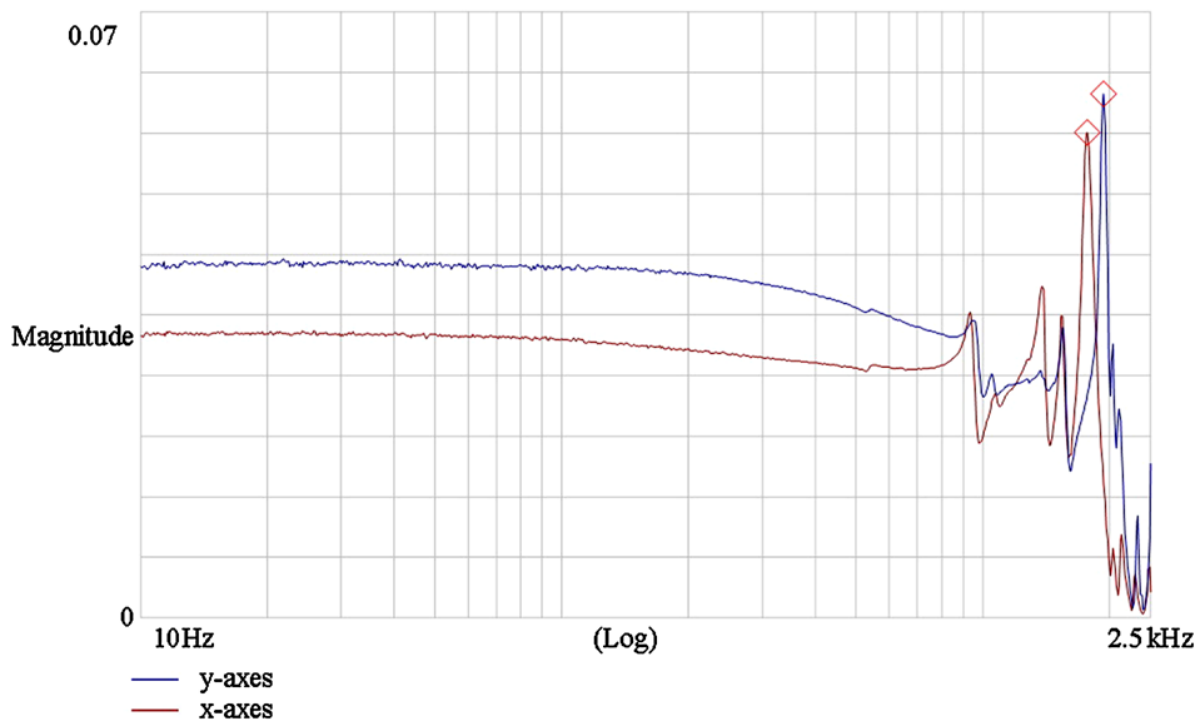

Figure 13 Excitation of the tip-tilt mirror as a function of the sinusoidal excitation frequency.

$7.5 \mathrm{~mm}$ (see Figure 12). The surface flatness $(75.9 \mathrm{~nm}$ peak-to-valley and $12.6 \mathrm{~nm}$ rms after attaching the piezos) is important because of its location close to the pupil image. Therefore, it is necessary to mount the mirror in a way that decouples the optical surface from differential thermal expansion of the mechanical parts and to reduce the mounting force as much as possible. The eigenfrequency of the whole system (mirror actuator and mounting) is about $1900 \mathrm{~Hz}$ (see Figure 13). At $1700 \mathrm{~Hz}$ closed-loop frequency the drive is operated in a minimum of excitation.

\subsection{Wave-Front Sensor Camera}

The WFS detector is an industrial DALSA 1M150 CMOS camera, which has the following properties and settings: 
- 8 bits per pixel;

- full well capacity $=200 \mathrm{ke}^{-}$;

- fill factor $\times$ quantum efficiency @ $500 \mathrm{~nm}=15 \%$;

- $1024 \times 1024$ pixels;

- region of interest $(\mathrm{ROI})=256 \times 224$ pixels;

- framerate $(\mathrm{ROI})=1700 \mathrm{~Hz}$;

- integration time $=100 \mu \mathrm{s}$;

- readout time $=380 \mu \mathrm{s}$;

- power consumption $=2 \mathrm{~W}$.

Since the camera is exposed to ca $80 \%$ of the full well capacity, the noise level (in $\mathrm{e}^{-}$) is dominated by shot noise and digitization noise is slightly less important. Readout noise is negligible. Thermal filler was inserted between the electronic components of the camera in order to guarantee conduction cooling at float altitude.

\section{Control Software}

This section deals with the CWS flight-control software as run on the embedded ARM micro-computer called CW-Com described in Section 4.2. The software communicates with the Instrument Control Unit (ICU), accepting commands either originating from the ICU itself or from the CWS Electronic Ground Support Equipment (EGSE). In return housekeeping data are sent to the ICU. The CW-Com also controls the behavior of the CW-AO computer ( $c f$. Section 4.2) by sending commands to it. Status information of the CW-AO computer is retrieved and forwarded as housekeeping to the ICU. The communication protocol between CW-Com and CW-AO is the same as at the GREGOR ground-based solar telescope (Volkmer et al., 2006). Furthermore, CW-Com communicates with the main telescope controller (MTC) and the pointing system (PS).

Section 5.1 describes the state-driven operation of the CWS as used during flight. Thereafter the behavior of the CWS in each specific state is discussed in Section 5.2, while Section 5.3 gives an overview of the communication between CW-Com and the MTC.

\subsection{State Driven Operation of CW-Com}

Due to the software design CWS is a state-driven instrument. The different states, the possible state transitions, and the behavior of the software within each state are described below.

Figure 14 illustrates the transitions between the seven possible states which are discussed in more detail below. Arrows with solid lines denote transitions that require the setstate command (either from the ICU or from an EGSE), while arrows with dotted lines represent state changes that are done automatically.

\subsection{States}

This section discusses each of the individual states CWS might adopt. The pre- and postconditions for each state are discussed. Furthermore, the behavior within each state is described here in detail. 
Figure 14 CWS state chart.

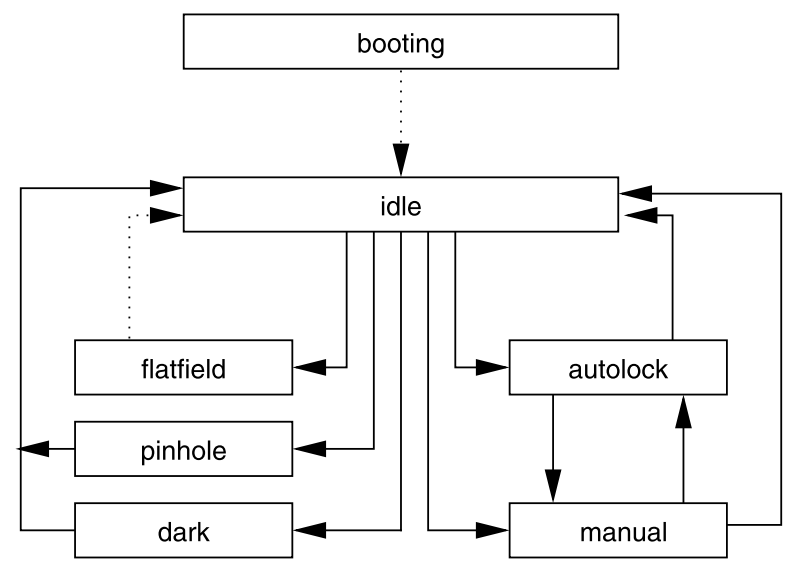

\subsection{1. booting}

The state booting is adopted once the CWS is powered up. While the CWS is in this state it does not accept any command; it will deliberately ignore each command trying to change the state. While the CWS is in this state, it will power up CoSM, the proximity electronics box, the camera and the CW-AO. It will remain in this state until CW-AO responds to the status command, i.e., provides the necessary data that enable the CW-Com to send reasonable HK data.

\subsection{2. idle}

This state is intended for manual operation of the CWS. The instrument will not take any action in this state except sending HK data. Whenever the idle state is reached, CW-Com will ramp the tip-tilt mirror and will make sure that the AO loop is open and F2 is set to field stop (light passes to the instruments). The CWS will accept any command including state transition commands in this state.

\subsection{3. flatfield}

In order to initiate a flatfield calibration, the ICU commands the CWS to the flatfield state. Although this state is called flatfield, it performs additional tasks. The CWS flatfielding procedure takes about 6 min and consists of the following steps:

i) ensure AO loop is open;

ii) adjustment of filter wheel for optimal light level;

iii) take the actual flatfield;

iv) set the F6 position to pinhole;

v) mark and measure the F6 reference spot positions of the Shack - Hartmann sensor that define the perfect wavefront;

vi) set F6 position to field.

The CWS does not accept state transition commands while it is in this state and will reach the idle state after completion of flatfielding. 


\subsection{4. pinhole}

Taking F2-pinhole images is also commanded by the ICU. CWS moves the tip-tilt mirror to its optical zero position and sets the F2 unit to the pinhole position. The minimum time CWS will remain in this state is $2 \mathrm{~s}$. While in this state, CWS does not accept state transition commands except to the idle state.

\subsection{5. dark}

If a setstate dark command is received, this state is adopted. CW-Com commands the F2 unit to the dark position before taking darks. The CWS requires about $10 \mathrm{~s}$ for the dark procedure to complete. It does not accept state transition commands while in this state and will only accept a transition command to the idle state after completion of the dark procedure.

\subsection{6. autolock}

This is the state intended for automated observations. A typical observation scenario looks like this:

i) point the telescope to the region of interest;

ii) take a flatfield (setstate flatfield);

iii) wait for all instruments to complete their flatfield;

iv) set CWS to autolock mode (setstate autolock);

v) wait for tip-tilt and focus loop to close (see field AO_runmode in HK of CWS);

vi) do actual observation sequence;

vii) open tip-tilt/focus loop by setting CWS to idle (setstate idle).

The behavior of the CWS in the autolock state is split into two parts, a startup phase that persists until a lock (tip-tilt plus focus) is achieved for the first time for at least $20 \mathrm{~s}$ (provided reasonable residuals are achieved) and an operational phase thereafter. During the startup phase a new reference image is taken before each attempt to close the loop. If the CWS loop crashes more than five times in a row or a lock signal from the PS is not received within $150 \mathrm{~s}$ during the operational phase (e.g. due to image motion beyond the range of the tip-tilt mirror), it will restart with a startup phase.

Note that the CWS will never leave the autolock state unless it receives a setstate idle or manual command. Especially if the CWS is not able to get a lock at the position the telescope points to (e.g. at the solar limb) it will remain in the startup phase of the autolock state forever. It is the responsibility of the ICU (or an operator of the EGSE) to monitor that a lock was achieved within a reasonable time.

\subsection{MTC Communication}

The main telescope controller (MTC) is physically connected to the CW-Com using an RS422 interface; hence, all communication between Sunrise and the telescope must pass CW-Com. All communication between the MTC and the ICU is forwarded by CW-Com transparently. The only commands sent to the MTC directly are positioning commands for the M2 mirror for focus and coma correction. 


\section{In-Flight Performance}

After the launch and opening of Sunrise's aperture door, the CWS was the first instrument to be powered up and put into operation. Figure 15 shows one of the first WFS images (left) and the corresponding correlation functions. The excellent quality of both image and correlation functions (compared to a ground-based solar SH-system) is obvious.

\subsection{Times of Closed Loop}

Figure 16 shows the number of closed-loop intervals as a function of the duration of the intervals. It can be seen that there is no time series longer than $45 \mathrm{~min}$. This is due to the fact that the gondola had problems stabilizing the telescope to within $\pm 45 \operatorname{arcsec}$ (range of the fast tip-tilt mirror) whenever shear winds occurred. Between Norway and Greenland, over the free ocean, wind gusts occurred less often than over Greenland or over the many coastlines during the last day. The lack of bandwidth of the gondola pointing system to correct wind gusts is one of the problems that were found during the flight. The overall observation time with image stabilization, i.e. without the observation overhead (flatfielding, calibrations, repointing etc.) was more than $33 \mathrm{~h}$.

\subsection{Residual Tip-Tilt Error}

A second problem showed up when analyzing the power spectrum of the residual tip-tilt error. Figure 17 shows the power spectrum for the azimuth (top) and elevation (bottom) as a

Figure 15 Sub-images and correlations functions of the wave-front sensor during closed-loop tracking. Screen shot taken from the flight-control system.

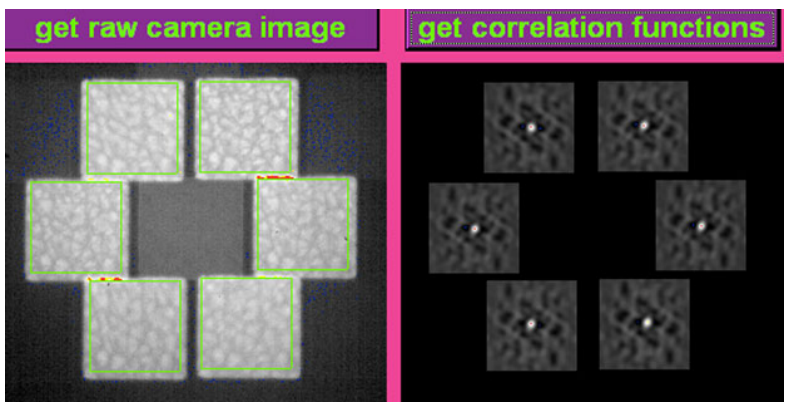

Number and duration (in min) of closed-loop intervals

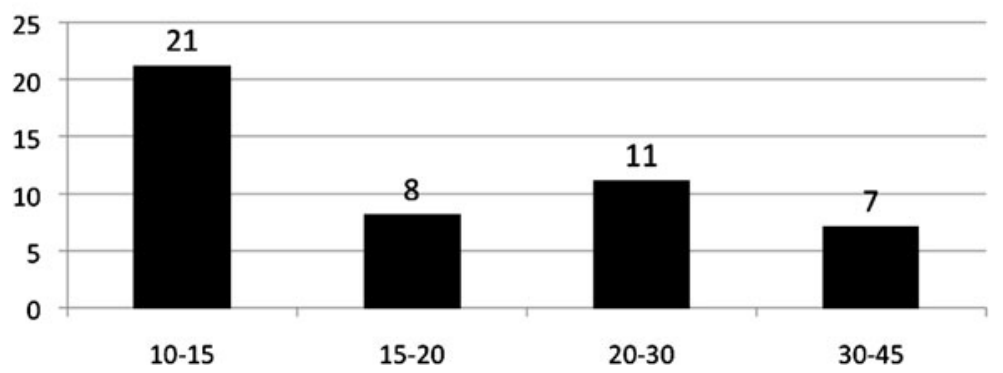

Figure 16 Number of closed-loop intervals longer than $10 \mathrm{~min}$. The total time with closed-loop observations was $33 \mathrm{~h}$. 
Figure 17 Power spectra of residual image jitter, for the azimuth (top panel), and elevation axis (bottom panel).
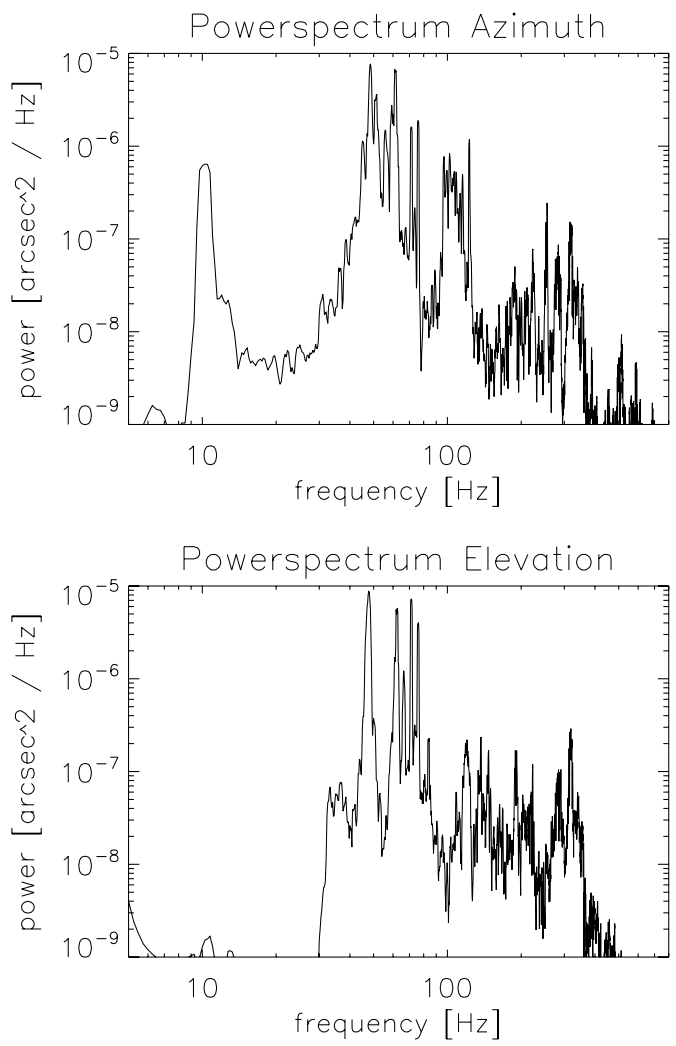

function of the frequency. It can be seen that vibrations in the frequency range of $30-90 \mathrm{~Hz}$ occur that were only partially damped (see Figure 6). Vibrations above $90 \mathrm{~Hz}$ are even amplified a bit. As a consequence, the residual image motion could not be reduced below $0.03-0.04^{\prime \prime} \mathrm{rms}$. The vibrations were most probably caused by the gondola. The telescope itself does not transmit frequencies below $30 \mathrm{~Hz}$ (with the exception of $10 \mathrm{~Hz}$ ), however, frequencies above $30 \mathrm{~Hz}$ could propagate from the gondola through the telescope. As a consequence, short exposure images (say, $30 \mathrm{~ms}$, as used in some SuFI wavelengths) have the same image quality as images that were exposed for $30 \mathrm{~s}$ (as used by IMaX and SuFI at $214 \mathrm{~nm})$.

For the next flight, vibration damping measures for the gondola have to be taken. Furthermore, it is possible to increase the tip-tilt bandwidth by about $70 \%$ by using only two $\mathrm{SH}$-sub-apertures instead of six during closed loop, allowing the detection of image shift and focus only. The resulting measurement accuracy will be reduced to $0.0035^{\prime \prime} \mathrm{rms}$. Changing between two and six sub-apertures can be done in flight, because it only requires a change of the parameter set.

\subsection{Focus Correction}

The in-flight focus correction proved to be vital. The uncorrected daily focus drift was $\pm 0.5 \lambda \mathrm{rms}$ between high and low solar elevations. By moving M2 in closed loop during observations, the focus error was reduced to $0.01 \lambda \mathrm{rms}$ at the CWS entrance focus. Phase 
diversity analysis of the flight data shows a differential focus of $\lambda / 20$ between SuFI and CWS and of $\lambda / 20$ between IMaX and CWS, well within the range to be corrected by using the phase diversity capabilities of SuFI and IMaX.

\section{Discussion}

During its successful flight in 2009, the Sunrise telescope had by far the highest pointing stability at the science focus ever achieved on a balloon-borne telescope. During its five day flight, it provided scientific data of seeing-free, unprecedented polarimetric accuracy and high resolution observations at UV wavelengths never observed before at similar resolution.

After the vibrations problems of the gondola have been solved, the goal of 0.005 arcsec rms pointing stability can be reached with the original six sub-aperture setup of the CWS. If some vibrations remain, the pointing could still be improved to ca. 0.01 arcsec rms by using the increased bandwidth of the two sub-aperture setup. The Sunrise team is therefore aiming for a second flight, ideally in 2012 close to the solar maximum.

Acknowledgements The German contribution to Sunrise is funded by the Bundesministerium für Wirtschaft und Technologie through Deutsches Zentrum für Luft- und Raumfahrt e.V. (DLR), Grant No. 50 OU 0401, and by the Innovationsfond of the President of the Max Planck Society (MPG). The Spanish contribution has been funded by the Spanish MICINN under projects ESP2006-13030-C06 and AYA200914105-C06 (including European FEDER funds). The HAO contribution was partly funded through NASA grant number NNX08AH38G.

Open Access This article is distributed under the terms of the Creative Commons Attribution Noncommercial License which permits any noncommercial use, distribution, and reproduction in any medium, provided the original author(s) and source are credited.

\section{References}

Barthol, P., Gandorfer, A., Solanki, S.K., Schüssler, M., Chares, B., Curdt, W., et al.: 2010, Solar Phys. doi:10.1007/s11207-010-9662-9.

Berkefeld, T.: 2007, In: Kneer, F., Puschmann, K.G., Wittmann, A.D. (eds.) Solar Facilities-Advanced Solar Science, Universitätsverlag Göttingen, Göttingen, 107.

Gandorfer, A., Grauf, B., Barthol, P., Riethmüller, T.L., Solanki, S.K., Chares, B., et al.: 2010, Solar Phys. doi:10.1007/s11207-010-9636-y.

Martínez Pillet, V., del Toro Iniesta, J.C., Álvarez-Herrero, A., Domingo, V., Bonet, J.A., González Fernández, L.: et al.: 2010, Solar Phys. doi:10.1007/s11207-010-9644-y.

Michau, V., Rousset, G., Fontanella, J.C.: 1992, In: Radick, R.R. (ed.) Real Time and Post Facto Solar Image Correction, Proc. NSO/Sacramento Peak Summer Workshop, National Solar Observatory, Sunspot, 124.

Schmidt, W., Solanki, S.K., Lites, B.W., Title, A.M., Martínez Pillet, V.: 2001, Astron. Nachr. $322,363$.

Schmidt, W., Berkefeld, T., Friedlein, R., Heidecke, F., Kentischer, T., von der Lühe, O.F., Sigwarth, M., Soltau, D., Wälde, E.: 2004, In: Oschmann, J.M. (ed.) Ground-based Telescopes, Proc. SPIE 5489, 1164.

Schmidt, W., Berkefeld, T., Feger, B., Friedlein, R., Gerber, K., Heidecke, F., et al.: 2006, In: Lewis, H., Bridger, A. (eds.) Advanced Software and Control for Astronomy, Proc. SPIE 6274, 62740H.

Schmidt, W., Solanki, S.K., Barthol, P., Berkefeld, T., Gandorfer, A., Knölker, M., Martínez Pillet, V., Schüssler, M., Title, A.: 2010, Astron. Nachr. 331, 601.

Solanki, S.K., Gandorfer, A.M., Schüssler, M., Curdt, W., Lites, B.W., Martinez-Pillet, V., Schmidt, W., Title, A.M.: 2003, In: Keil, S.L., Avakyan, S.V. (eds.) Innovative Telescopes and Instrumentation for Solar Astrophysics, Proc. SPIE 4853, 129. 
Volkmer, R., von der Lühe, O., Kneer, F., Staude, J., Hofmann, A., Schmidt, W., et al.: 2003, In: Keil, S.L., Avakyan, S.V. (eds.) Innovative Telescopes and Instrumentation for Solar Astrophysics, Proc. SPIE 4853, 360 .

Volkmer, R., von der Lühe, O., Kneer, F., Staude, J., Berkefeld, T., Caligari, P., et al.: 2006, In: Stepp, L.M. (ed.) Ground-Based and Airborne Telescopes, Proc. SPIE 6267, 62670W.

von der Lühe, O., Soltau, D., Berkefeld, T., Schelenz, T.: 2003, In: Keil, S.L., Avakyan, S.V. (eds.) Innovative Telescopes and Instrumentation for Solar Astrophysics, Proc. SPIE 4853, 187. 